8. The linear dependence of functions of several variables and certain completely integrable systems of partial differential equations. Transactions of the American Mathematical Society, vol. 17, No. 4, pp. 483-516, October.

9. On the linear dependence of functions of one variable. Bulletin of the American Mathematical Society, vol. 23, No. 3, pp. 118-122, December.

1917.

10. On the general theory of curved surfaces and rectilinear congruences. Proceedings of the National Academy of Sciences, vol. 3, No. 10, pp. 587-592, October.

11. Some geometric characterizations of isothermal nets on a curved surface. Transactions of the American Mathematical Society, vol. 18, No. 4, pp. 480-488, October.

1918.

12. Note on conjugate nets with equal point invariants. Bulletin of the American Mathematical Society, vol. 24, No. 5, pp. 221-225, February.

13. The intersections of a straight line and hyperquadric. Annals of Mathematics, ser. 2, vol. 19, No. 3, pp. 207-209, March.

14. Plane nets with equal invariants. Annals of Mathematics, ser. 2, vol. 19, No. 4, pp. 246-250, June.

15. On certain projective generalizations of metric theorems and the curves of Darboux and Segre. Proceedings of the National Academy of Sciences, vol. 4, No. 11, pp. 346-349, November.

1919.

16. Memoir on the general theory of surfaces and rectilinear congruences. Transactions of the American Mathematical Society, vol. 20, No. 2, pp. 79-153, April.

\title{
REDUCTION OF THE ELLIPTIC ELEMENT TO THE WEIERSTRASS FORM.
}

BY PROFESSOR F. H. SAFFORD.

(Read before the American Mathematical Society April 26, 1919.)

IN Enneper's Elliptische Functionen, page 27, may be found a method due to Weierstrass of reducing the general elliptic element to the Weierstrass form. Briefly, from

$\sqrt{R\left(x_{0}\right)} \sqrt{\left(4 s^{3}-g_{2} s-g_{3}\right)}$
$+\frac{1}{2} R^{\prime}\left(x_{0}\right)\left[s-\frac{1}{24} R^{\prime \prime}\left(x_{0}\right)\right]+\frac{1}{24} R\left(x_{0}\right) R^{\prime \prime \prime}\left(x_{0}\right)$
$2\left[s-\frac{1}{24} R^{\prime \prime}\left(x_{0}\right)\right]^{2}-\frac{1}{2} A \cdot R\left(x_{0}\right)$ 
comes

$$
\frac{d x}{\sqrt{R(x)}}=\frac{-d s}{\sqrt{\left(4 s^{3}-g_{2} s-g_{3}\right)}}=d u .
$$

Enneper adds: “Diese Resultate wurden zuerst veröffentlicht von W. Biermann: 'Problemata quædam mechanica functionum ellipticarum ope soluta,' Diss. Berlin, 1864,"' and the latter states that they were given in Weierstrass's lectures.

Necessary additional formulas are

$$
\begin{aligned}
& R(x)=A x^{4}+4 B x^{3}+6 C x^{2}+4 B^{\prime} x+A^{\prime} \\
& =r_{0}+r_{1}\left(x-x_{0}\right)+r_{2}\left(x-x_{0}\right)^{2}+r_{3}\left(x-x_{0}\right)^{3} \\
& +r_{4}\left(x-x_{0}\right)^{4} \\
& r_{0}=R\left(x_{0}\right), \quad r_{1}=R^{\prime}\left(x_{0}\right), \quad r_{2}=R^{\prime \prime}\left(x_{0}\right) / 2 \text {, } \\
& r_{3}=R^{\prime \prime \prime}\left(x_{0}\right) / 6, \quad r_{4}=R^{\prime \prime \prime \prime}\left(x_{0}\right) / 24, \\
& g_{2}=A A^{\prime}+3 C^{2}-4 B B^{\prime}=r_{0} r_{4}-\frac{1}{4} r_{1} r_{3}+\frac{1}{12} r_{2}^{2} \\
& A C A^{\prime}+2 B C B^{\prime}-A B^{\prime 2}-A^{\prime} B^{2}=4 \epsilon_{1} \epsilon_{2} \epsilon_{3} \text {, } \\
& =\frac{1}{6} r_{0} r_{2} r_{4}+\frac{1}{4} r_{1} r_{2} r_{3}-\frac{1}{16} r_{0} r_{3}^{2}-\frac{1}{16} r_{4} r_{1}^{2}-\frac{1}{2} \frac{1}{16} r_{2}^{3} \\
& =-4\left(\epsilon_{1} \epsilon_{2}+\epsilon_{2} \epsilon_{3}+\epsilon_{3} \epsilon_{1}\right) \text {, } \\
& s=\wp(u+c), \quad\left(x_{0} \text { and } c \text { are arbitrary constants }\right) .
\end{aligned}
$$

Enneper later uses (1) in obtaining the addition formula for $\wp(u+v)$, and Greenhill also quotes (1), adding a brief note.

Haentzschel, in Reduction der Potentialgleichung, discussing surfaces of revolution in a potential problem, obtains, following Wangerin, this differential equation

(4) $F^{\prime 2}(u)=A F^{4}(u)+4 B F^{3}(u)+6 C F^{2}(u)$

for which he gives a solution

$$
+4 B^{\prime} F(u)+A^{\prime}=R(F),
$$

$$
x=F(u)=\alpha+\frac{R^{\prime}(\alpha) / 4}{s-R^{\prime \prime}(\alpha) / 24},
$$


which is obtainable from (1) by using for $x_{0}$ the value $\alpha$, any root of $R(x)=0$. Haentzschel adds “. . . so könnte nach Herrn Weierstrass der Ausdruck (5) doch noch nicht als die allgemeinste Form des Integrals von (4) gelten, sondern als solchen leitet Herr Weierstrass ab," and then gives (1) above. Haentzschel uses the simpler solution in obtaining various families of orthogonal curves according to the methods of conjugate functions, but merely gives an outline for the case of the (apparently) more general solution.

The writer has given discussions and extensions of Haentzschel's results in the Archiv der Mathematik und Physik. In addition to obtaining the equations of the curves corresponding to the more general solution, he has shown that these equations are factorable and include the curves from the simpler case. These results, omitting computations in general, have appeared in this Bulletin, June, 1899, March, 1912, November, 1917.

The object of the present paper is to show that solution (1) arises solely from a particular choice of constants in the simpler form (5). But (5) is itself of considerable interest because it may be used to obtain the equation referred to by Durège as "Die Landensche Substitution." If $R(x)$ be replaced by Legendre's form, (5) gives

$$
x=\operatorname{sn}(u \sqrt{e})=\sqrt{(e)\left(s-\epsilon_{1}\right) /\left(s-\epsilon_{2}\right)\left(s-\epsilon_{3}\right)},
$$

while the commonly quoted relation between sn and $\wp$ is

$$
\operatorname{sn}(u \sqrt{e})=\sqrt{e} / \sqrt{\left(t-a_{3}\right)},
$$

in which $t$ and $a_{3}$ correspond respectively to $s$ and $\epsilon_{3}$; in both formulas $e$ is essentially $A^{\prime}$ used above. From (6) and (7) the substitution may be derived. Proceeding to the more important derivations, the fundamental equation (1) is solved for $s$, giving

$$
\begin{aligned}
s & =\wp(u+c) \\
& =\frac{\sqrt{r_{0}} \sqrt{R(x)}+r_{0}+r_{1}\left(x-x_{0}\right) / 2+r_{2}\left(x-x_{0}\right)^{2} / 6}{2\left(x-x_{0}\right)^{2}}
\end{aligned}
$$

Next let $x_{0}$ have the value $\alpha$, where $\alpha$ is a root of $R(x)=0$, so that the corresponding form of (8) is 


$$
\begin{aligned}
s_{1} & =\wp\left(u+c_{1}\right) \\
& =\frac{t_{0}+t_{1}(x-\alpha) / 2+t_{2}(x-\alpha)^{2} / 6}{2(x-\alpha)^{2}} \\
& =\frac{t_{1} / 4+t_{2}(x-\alpha) / 12}{x-\alpha} .
\end{aligned}
$$

It should be noted that in (9)

$$
t_{0}=R(\alpha)=0, \quad t_{1}=R^{\prime}(\alpha), \quad t_{2}=R^{\prime \prime}(\alpha) / 2 .
$$

As $c$ and $c_{1}$ are merely constants of integration in solutions of the same differential equation, it is essential to show what relation between them will make the two solutions identical. By the aid of the usual formula

$$
\wp(u \pm v)=\frac{[\wp(u)+\wp(v)]\left[4 \wp(u) \wp(v)-g_{2}\right]}{-2 g_{3} \mp 2 \wp^{\prime}(u) \wp^{\prime}(v)}
$$

from (8) and (9) follows

$$
c-c_{1}=\wp^{-1}(s)-\wp^{-1}\left(s_{1}\right)
$$

or

$$
s_{2}=\wp\left(c-c_{1}\right)=\frac{\left(s+s_{1}\right)\left(4 s s_{1}-g_{2}\right)-2 g_{3}-2 s^{\prime} s_{1}{ }^{\prime}}{4\left(s-s_{1}\right)^{2}} .
$$

The direct reduction of the second member of (13) would be extremely long, but it can be readily carried far enough by computing the coefficients of certain powers of $x$ to show that

$$
s_{2}=\wp\left(c-c_{1}\right)=\frac{r_{0}+r_{1}\left(\alpha-x_{0}\right)}{2\left(\alpha-r_{0}\right)^{2}} \frac{\left.2+x_{0}\right)^{2} / 6}{-},
$$

which is the desired relation mentioned above. It is important to notice that $s_{2}$ is obtainable from $s$ by replacing $x$ by $\alpha$, while $s_{1}$ was similarly obtained by replacing $x_{0}$ by $\alpha$. In both cases the radicals in $s$ vanish. Though not necessary, a verification of the preceding result, (14), may be effected without difficulty with the aid of (11) and (12) by computing

$$
s=\wp\left[\wp^{-1}\left(s_{1}\right)+\wp^{-1}\left(s_{2}\right)\right]=\wp(u+c) .
$$

Thus (8) or its equivalent form, (1), the fundamental formula due to Weierstrass, is obtainable directly from the simple solution (5) by a suitable choice of the constant $c$ as given by (14).

University of Pennsylvania, Philadelphia, Pa. 